\title{
Expression and enzyme activity of glutathione reductase is upregulated by Fe-deficiency in graminaceous plants
}

\author{
Khurram Bashir - Seiji Nagasaka - Reiko Nakanishi Itai - Takanori Kobayashi • \\ Michiko Takahashi · Hiromi Nakanishi · Satoshi Mori · Naoko K. Nishizawa
}

Received: 7 November 2006/Accepted: 23 July 2007/Published online: 21 August 2007

(C) Springer Science+Business Media B.V. 2007

\begin{abstract}
Glutathione reductase (GR) plays an important role in the response to biotic and abiotic stresses in plants. We studied the expression patterns and enzyme activities of GR in graminaceous plants under Fe-sufficient and $\mathrm{Fe}$ deficient conditions by isolating cDNA clones for chloroplastic GR $(H v G R l)$ and cytosolic GR $(H v G R 2)$ from barley. We found that the sequences of GR1 and GR2 were highly conserved in graminaceous plants. Based on their nucleotide sequences, $H v G R 1$ and $H v G R 2$ were predicted to encode polypeptides of 550 and 497 amino acids, respectively. Both proteins showed in vitro GR activity, and the specific activity for HvGR1 was 3-fold that of HvGR2. Northern blot analyses were performed to examine the expression patterns of $G R 1$ and $G R 2$ in rice $(O s)$, wheat $(T a)$, barley $(H v)$, and maize $(Z m) . H v G R 1, H v G R 2$, and $T a G R 2$ were upregulated in response to Fe-deficiency. Moreover, $H v G R l$ and TaGRl were mainly expressed in shoot tissues, whereas $H v G R 2$ and TaGR2 were primarily observed in root tissues. The GR activity increased in roots
\end{abstract}

Khurram Bashir and Seiji Nagasaka contributed equally to this work. The nucleotide sequences reported in this paper have been submitted to the DDBJ/GenBankTM/EBI Data Bank with accession numbers AB277096 and AB277097 for $H v G R 1$ and $H v G R 2$, respectively.

K. Bashir $\cdot$ S. Nagasaka $\cdot$ R. N. Itai · T. Kobayashi ·

M. Takahashi · H. Nakanishi · S. Mori · N. K. Nishizawa ( $\square)$ Laboratory of Plant Biotechnology, Department of Global Agricultural Sciences, Graduate School of Agricultural and Life Sciences, The University of Tokyo, 1-1-1 Yayoi, Bunkyo-Ku, Tokyo 113-8657, Japan

e-mail: annaoko@mail.ecc.u-tokyo.ac.jp

S. Nagasaka - R. N. Itai - T. Kobayashi · N. K. Nishizawa

Core Research for Evolutional Science and Technology

Corporation (CREST), Japan Science and Technology

Corporation (JST), Kawaguchi-Shi, Saitama, Japan of barley, wheat, and maize and shoot tissues of rice, barley, and maize in response to Fe-deficiency. Furthermore, it appeared that GR was not post-transcriptionally regulated, at least in rice, wheat, and barley. These results suggest that GR may play a role in the Fe-deficiency response in graminaceous plants.

Keywords Barley · Redox system - Glutathione · Glutathione reductase - Graminaceous plants .

Fe-deficiency

$\begin{array}{ll}\text { Abbreviations } \\ \text { GSH } & \text { Glutathione } \\ \text { GR } & \text { Glutathione reductase } \\ \text { GR1 } & \text { Chloroplastic GR } \\ \text { GR2 } & \text { Cytosolic GR } \\ \text { Fe } & \text { Iron }\end{array}$

\section{Introduction}

The tripeptide glutathione ( $\gamma$-Glu-Cys-Gly; GSH) is the major non-protein thiol compound in eukaryotic cells and participates in the regulation of cellular redox status. Essential for plant growth and development, this antioxidant is a key cellular redox component that functions in the regulation of gene expression and the cell cycle (Noctor et al. 2002). This is particularly important in plants during exposure to oxidative stress, when reactive oxygen species (ROS) are formed and GSH operates as a central component of the ascorbic acid-GSH cycle (Noctor and Foyer 1998). Other functions of GSH include the storage and long-distance transport of reduced sulfur (Brunold and Rennenberg 1997), conjugation with secondary plant metabolites and xenobiotics via glutathione S-transferases 
(Marrs 1996; Alfenito et al. 1998), and the formation of phytochelatins in response to heavy metal exposure (Cobbett 2000). Important developmental processes such as root meristem activity (Vernoux et al. 2000) and flowering (Ogawa et al. 2001) are also regulated by GSH levels.

GSH must be in its reduced form to fulfill many of its roles. This is particularly true when it acts as antioxidant against ROS such as hydrogen peroxide and super oxide (Noctor and Foyer 1998). Thus, the enzyme glutathione reductase (GR) is essential for the functioning of GSH and is a prevalent member of the flavoprotein oxidoreductase family in both eukaryotes and prokaryotes. GR catalyzes the reaction that converts oxidized glutathione (GSSG) to reduced glutathione $(\mathrm{GSH})$ using $\mathrm{NAD}(\mathrm{P}) \mathrm{H}$ as an electron donor (Meister 1988). In plants, GR plays a key role in the response to oxidative stress by maintaining the intracellular pool of GSH. Expression of GR is upregulated under stresses such as changes in salinity, drought, high light intensity, mechanical wounding, chilling, and exposure to heavy metals and herbicides (Foyer et al. 1991; Mullineaux and Creissen 1997; Apel and Hirt 2004; Romero-Puertas et al. 2006).

Iron $(\mathrm{Fe})$ is an essential element in plants, and is required for cellular events such as respiration, chlorophyll biosynthesis, and photosynthetic electron transport. Fe is required for the synthesis of heme and chlorophyll. Indeed, low chlorophyll content (chlorosis) of young leaves is the most obvious visible symptom of Fe-deficiency. A number of heme and non-heme proteins involved in redox systems also require $\mathrm{Fe}$. The most well-known heme proteins are cytochromes, which contain $\mathrm{Fe}$ as a prosthetic group. Other heme enzymes such as catalase and peroxidase also depend on Fe for their activity. In non-heme proteins such as those containing a $\mathrm{Fe}-\mathrm{S}$ domain, $\mathrm{Fe}$ associates with the thiol group of cysteine, with inorganic sulfur in clusters, or with both, such as in ferredoxin. Isozymes of superoxide dismutases (SOD), which detoxify superoxide anions, also require $\mathrm{Fe}$ as a prosthetic group. The activities of catalase, peroxidase (Iturbe-Ormaetxe et al. 1995; Tewari et al. 2005), and ascorbate peroxidase (Iturbe-Ormaetxe et al. 1995; Ishikawa et al. 2003; Zaharieva et al. 2004; Tewari et al. 2005) decrease under Fe-deficient conditions, whereas the activities of different isozymes of SOD increase (Tewari et al. 2005). These enzymes play a role in scavenging ROS; thus Fe-deficiency, like Zn-deficiency (Cakmak and Marschner 1988), can trigger secondary oxidative stress.

Although Fe is abundant in soil, it is mainly present in oxidized Fe(III) compounds, which are poorly soluble in neutral to alkaline soils. Calcareous soils account for $30 \%$ of the world's cultivated soil (Chen and Barak 1982). As such, Fe-deficiency is a worldwide problem that is responsible for serious reductions in crop yields. The molecular basis of Fe-acquisition from soil has been identified and strategies have been proposed to overcome this problem (For review see: Curie and Briat 2003; Mori 2001). Graminaceous plants secrete a family of small molecules called mugineic acid family phytosiderophores (MAs), to solubilize soil Fe (Takagi 1976). The Fe(III)MAs complex is readily absorbed by the plant. DMA, synthesized by DMA synthase (DMAS; Bashir et al. 2006), is the first MA synthesized in the MA biosynthetic pathway and the subsequent steps differ depending on the plant species or even the cultivar (Ma et al. 1999; Mori 2001). The production and secretion of MAs markedly increases in response to Fe deficiency, and tolerance to Fe deficiency in graminaceous plants is strongly correlated with MAs secreted. Although excess $\mathrm{Fe}$ is known to trigger oxidative stress (Halliwell and Gutteridge 1986), little is known about the role of Fe-deficiency-induced oxidative stress and the role of antioxidants such as GSH in plant responses to Fe-deficiency. Therefore, understanding the mechanisms involved in the Fe-deficiency response is of extreme importance to create Fe-deficiency-tolerant plants and to thereby increase crop production.

The expression of GR is regulated by a variety of biotic and abiotic stresses; however, to date no report has described the expression pattern of GR in response to $\mathrm{Fe}$ deficiency stress in plants. These responses are especially of interest in barley, which is one of the most Fe-deficiency-tolerant species among graminaceous crops. To investigate the regulation of GR in graminaceous plants, cytosolic and chloroplastic isoforms were cloned from barley and their expression patterns as well as enzyme activity were examined under Fe-sufficient and Fe-deficient conditions in graminaceous plants.

\section{Materials and methods}

\section{Cloning of $H v G R 1$ and $H V G R 2$}

A full-length cDNA library prepared from Fe-deficient barley roots (Higuchi et al. 2001) was screened to clone the full-length cDNA of barley cytosolic GR (HvGR2). The GenBank accession AB063249, having homology with OsGR2 (LOC_Os02g56850) was previously isolated in an attempt to clone DMAS. This clone is shorter than OsGR2 and consists of about $70 \%$ of the sequence of OsGR2. ORF of $\mathrm{AB} 063249$ was labeled with ${ }^{32} \mathrm{P}$, and approximately 200,000 colonies of the cDNA library were screened through colony hybridization as described previously (Higuchi et al. 2001).

Partial sequences of barley chloroplastic GR ( $H v G R l)$ were identified as unigene numbers 7437 and 37686 in the 
HarvEST database (Version 1.47; http://www.harvest. ucr.edu/) because of their strong homology to rice chloroplastic GR (OsGR1; LOC_Os03g06740). The HvGRl ORF was amplified from the cDNA library using the $5^{\prime}$-CACCATGGCGACAACCGCGGCCCTCCC- $3^{\prime}$ and $5^{\prime}$-TACTT CTGAGCGACGACCTCATC- $3^{\prime}$ as forward and reverse primers, respectively. The amplified cDNA clone was subcloned into pENTR/D-TOPO (Invitrogen, Carlsbad, CA) and sequenced using a Thermo Sequenase Cycle Sequencing Kit (Shimadzu, Kyoto, Japan) and a DSQ-2000L DNA sequencer (Shimadzu).

The rice genome database (http://www.tigr.org/) was searched to determine the number and location of GR genes in the rice genome. The homologs of GR in different crops were identified through BLAST (http://www.ncbi. nlm.nih.gov/BLAST/), and phylogenetic tree was constructed using CLUSTAL W Neighbor-Joining method and the tree was visualized with TreeView.

\section{Plant material}

Seeds of barley (Hordeum vulgare L. cv. Ehimehadaka no. 1), wheat (Triticum aestivum L. cv. Chinese spring), maize (Zea mays cv. Alice), and rice (Oryza sativa L. cv. Nipponbare) were germinated on wet filter paper at room temperature. After germination, the seedlings were transferred to a 20-L plastic container containing a nutrient solution with the following composition: $0.7 \mathrm{mM} \mathrm{K}_{2} \mathrm{SO}_{4}$, $0.1 \mathrm{mM} \mathrm{KCl}, \quad 0.1 \mathrm{mM} \mathrm{KH}_{2} \mathrm{PO}_{4}, 2.0 \mathrm{mM} \quad \mathrm{Ca}\left(\mathrm{NO}_{3}\right)_{2}$, $0.5 \mathrm{mM} \mathrm{MgSO}, 10 \mathrm{M} \mathrm{H}_{3} \mathrm{BO}_{3}, 0.5 \mu \mathrm{M} \mathrm{MnSO}_{4}, 0.2 \mu \mathrm{M}$ $\mathrm{CuSO}_{4}, 0.5 \mu \mathrm{M} \mathrm{ZnSO}{ }_{4}, 0.05 \mu \mathrm{M} \mathrm{Na} \mathrm{MoO}_{4}$, and $0.1 \mathrm{mM}$ Fe-EDTA for rice, while the nutrient solution for the maize, barley and wheat was prepared as described (Kanazawa et al. 1994). The $\mathrm{pH}$ of nutrient solution was adjusted daily to 5.5 with $1 \mathrm{M} \mathrm{HCl}$ and was renewed weekly. For the Fe-deficiency treatments, 2-week-old plants were transferred to a nutrient solution without $\mathrm{Fe}$ and grown for 2 week. Symptoms of Fe-deficiency were apparent after 2 weeks, at which point the roots and leaves were harvested, frozen in liquid nitrogen, and stored at $-80^{\circ} \mathrm{C}$ until use.

Northern blot analysis

Total RNA was extracted from roots and shoots, and $10 \mu \mathrm{g}$ were electrophoresed in $1.2 \%(\mathrm{w} / \mathrm{v})$ agarose gels containing $0.66 \mathrm{M}$ formaldehyde and transferred to Hybond-N+ membranes (Amersham, Piscataway, NJ). For GR1, the full length ORF of $H v G R l$ was labeled with digoxigenin (DIG) and used as a probe for Northern blot analysis. For GR2, Northern blot analysis was performed with full length ORF of $H v G R 2$ labeled with DIG. For $O s G R 2$, a full length ORF was cloned from a rice cDNA library with the forward and reverse primers $5^{\prime}$-ATGGCTAGGAAGATGCTCAAGG-3' and 5'-CTACAAGTTTGTCTTTGGCTTGG-3', then sequenced using a Thermo Sequenase Cycle Sequencing Kit (Shimadzu) and a DSQ-2000L DNA sequencer (Shimadzu), and labeled with DIG. Each probe was individually incubated with each membrane at $68^{\circ} \mathrm{C}$ and processed as described previously (Engler-Blum et al. 1993; Yoshihara et al. 2003).

\section{Determination of GR activity}

To subclone $H v G R 1$ into pMAL-c2 (New England Biolabs, Beverly, MA), the ORF was amplified with primers such that it contained an XbaI site at the $5^{\prime}$ end and a HindIII site at the $3^{\prime}$ end. The ORF was amplified, subcloned into pCR-Blunt II-TOPO (Invitrogen, Carlsbad, CA), and sequenced. The resulting plasmid was then digested with $\mathrm{XbaI}$ and HindIII and the excised fragment containing $H v G R l$ was subcloned into pMAL-c2. This plasmid was designated pMAL-c2$H v G R 1 . H v G R 2$ and AB063249 were also subcloned into pMAL-c2 in the same manner. These pMAL-c2 plasmids were introduced into $E$. coli XL1-Blue, which were induced to produce the recombinant fusion proteins. The proteins were purified as described by Higuchi et al. (1999).

The GR activity was determined using $1 \mu \mathrm{g}$ of recombinant HvGR1, HvGR2, and AB063249 bound to a maltose-binding protein with a GR assay kit (Trevigen, Gaithersburg, MD) according to the manufacturer's instructions. To determine the effect of Fe-deficiency on total GR activity in rice, wheat, barley, and maize, the crude extract from plants grown under Fe-sufficient and Fe-deficient conditions was extracted using the buffer provided with the kit according to the manufacturer's instructions (Trevigen). The proteins were quantified using the Bradford assay (Bradford 1976) and diluted to $1 \mu \mathrm{g} \mu \mathrm{l}^{-1}$ with dilution buffer. Each assay was performed at least in duplicate with $40 \mu \mathrm{g}$ of total protein.

\section{Results}

Cloning of $H v G R 1$ and $H v G R 2$

The GenBank cDNA clone AB063249 is highly homologous to rice and wheat GR. We screened the full-length cDNA library using AB063249 as a probe and isolated two cDNA clones: one corresponding to AB063249 and one for cytosolic GR (HvGR2). The $H v G R 2$ contained the full length of AB063249 with some variation at the $5^{\prime}$ UTR region. The chloroplast GR $(H v G R l)$ was cloned with 
primers designed to amplify the HvGRl ORF. The sequence of GR was highly conserved among graminaceous plants and $H v G R 2$ showed high homology to wheat (Triticum monococum; 96\%) and rice (89\%) cytosolic GR (Fig. 1). Likewise, $H v G R 1$ showed $86 \%$ homology to rice chloroplastic GR. Three genes for GR exist in rice (Fig. 1). The gene for cytosolic GR (OsGR2) is located on rice chromosome 2 and is split into 16 exons (http://www.tigr. org/), whereas rice chloroplastic GR (OsGRl) is located on chromosome 3 and split into 10 exons. Rice was also found to contain a partial sequence for GR1 (LOC_Os10g28000) located on chromosome 10. This sequence lacked a FADbinding domain and part of the NADPH-binding domain.

Based on their nucleotide sequences, $H v G R l$ and $H v G R 2$ were predicted to encode polypeptides of 550 and 497 amino acids, respectively (Fig. 2). The NADPH

(A)
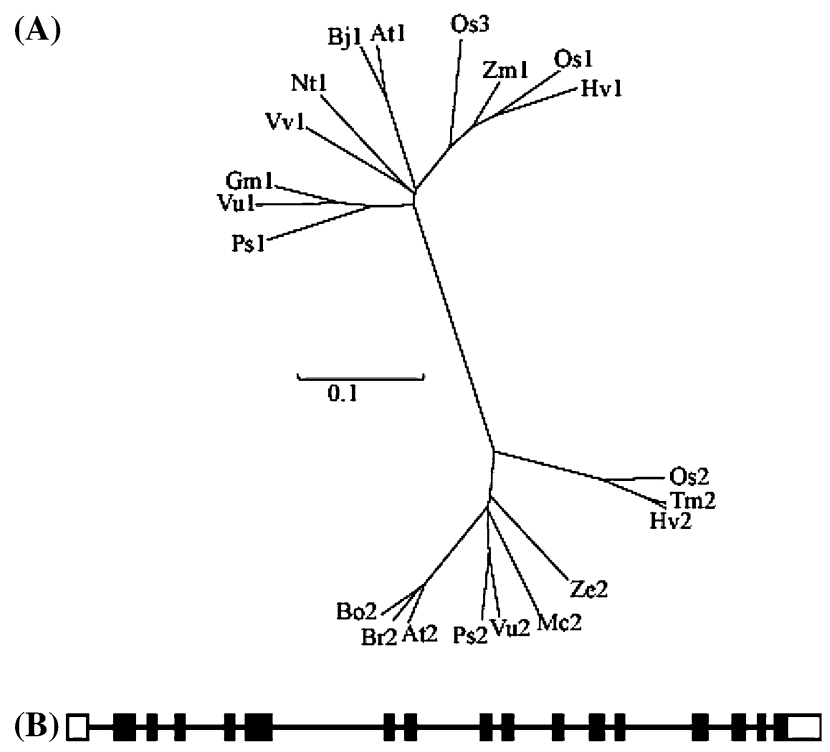

(C)

(D)

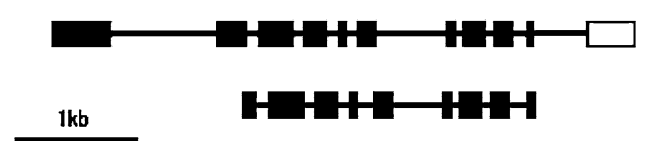

Fig. 1 Phylogeny and genomic structure of GR. (a) Unrooted phylogenic tree of GR. Hv, Hordeum vulgare (Hv1: AB277096; Hv2: AB277097); Tm, T. monococum (Tm2: AY364467); Os, Oryza sativa (Os1: AK066398; Os2: AK103418; Os3: AK108799); Zm, Zea mays (Zm1: AJ006055); Bo, Brassica oleracea (Bo2: AB125639); Br, Brassica rapa (Br2: AF008441); At, Arabidopsis thaliana (At1: AY054677; At2: AF360228); Ze, Zinnia elegans (Ze2: AB158513); Ps, Pisum sativum (Ps1: X90996; Ps2: Q43621); Mc, Mesembryanthemum crystallinum (Mc2: AJ400816); Nt, Nicotiana tabacum (Nt1: $\mathrm{P} 80461)$; Vv, Vitis vinifera (Vv1: AF019907); Bj, Brassica juncea (Bj1: AF349449). Vu, Vigna unguiculata (Vu1: DQ267474; Vu2: DQ267475); Gm, Glycine $\max$ (Gm1: P48640); GR1 represents chloroplastic while GR2 represents cytosolic GR. (b) Genomic structure of rice cytosolic GR (OsGR2). (c) Genomic structure of rice chloroplastic GR (OsGR1). (d) Genomic structure of rice partial chloroplastic GR (OsGR3). - , Introns; $\mathbf{\square}$, Exons; $\square$, Untranslated regions and substrate-binding domains were identified by aligning the sequences with those of other GRs that had been characterized previously. Similar to other GRs, these domains were conserved for graminaceous $G R 1$ and $G R 2$.

\section{Enzyme activity}

$H v G R 1$ and $H v G R 2$ were expressed in E. coli as a maltosebinding fusion protein, and purified protein was tested for the ability to reduce GSSG. AB063249 was also expressed as a maltose-binding protein; however, it lacked a complete FAD-binding domain and part of the substrate-binding domain, and was therefore used as a negative control. HvGR1 and HvGR2 were shown to catalyze the conversion of GSSG to GSH (Fig. 3) as measured by the decrease in absorbance of NADPH at $340 \mathrm{~nm}$. The enzyme activity for HvGR1 was three times higher than that of HvGR2.

The expression of $G R 1$ and $G R 2$ under Fe-deficient conditions

To confirm the Fe-deficient status of the plants the expression of DMAS, a gene essential for Fe-deficiency response in graminaceous plants, was observed through Northern and Western blot analysis. Northern blot analysis confirmed that the expression of DMAS in all species examined is upregulated in response to Fe-deficiency (Bashir et al. 2006). The results of Western blot analysis were also in line to support the Fe-deficient status of plants (data not shown). Northern blot analyses confirmed that AB063249 did not express under Fe-sufficient or deficient conditions (data not shown). Further, Northern blot analyses were performed to determine whether the expression of GR1 and GR2 was upregulated under Fe-deficient conditions in graminaceous crops. The expression of both genes was upregulated in barley in response to Fe-deficiency (Fig. 4). In addition, the expression of TaGRl was not elevated in response to Fe-deficiency, while that of TaGR2 was (Fig. 4). No band was detected for maize, and faint bands were detected for rice when $H v G R 2$ was used as probe (data not shown). Northern blot analysis was also performed using $O s G R 2$ as a probe, which showed that the expression of $O s G R 2$ decreased slightly under Fe-deficient conditions (Fig. 4b). The expression of $H v G R l$ was mainly localized to shoot tissues and only slight expression was observed in roots. Conversely, the expression of $H v G R 2$ was mainly observed in roots, where it was significantly upregulated in response to Fe-deficiency. The expression of $H v G R 2$ was also upregulated in response to $\mathrm{Zn}$-deficiency in both root and shoot tissues (data not shown). Although we did not attempt a Northern blot analysis using OsGRI 
Fig. 2 Sequence homology between rice and barley GR. \#, GSSG-binding domain; *, NAD(P)H-binding domain; +, FAD-binding domain

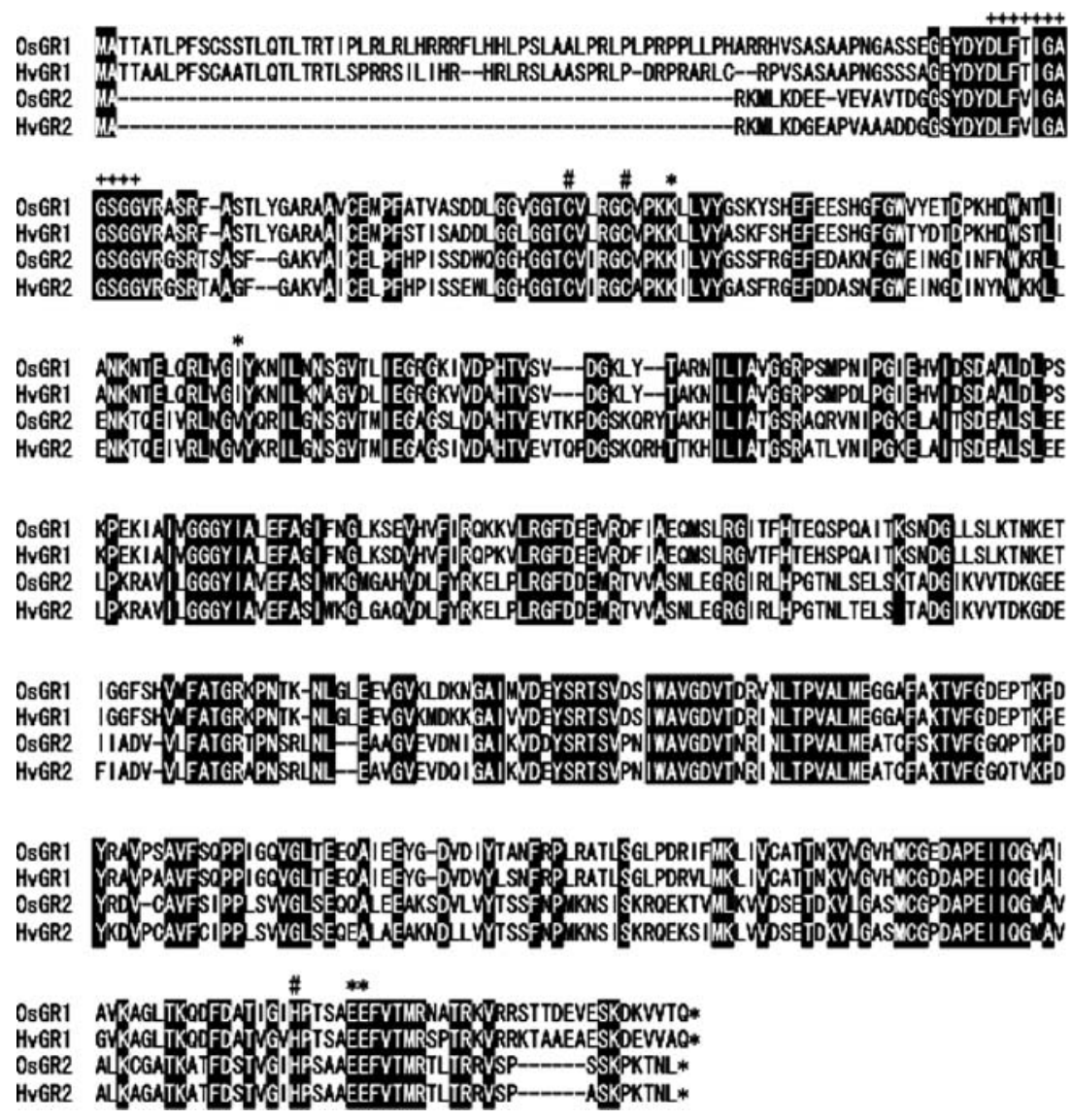

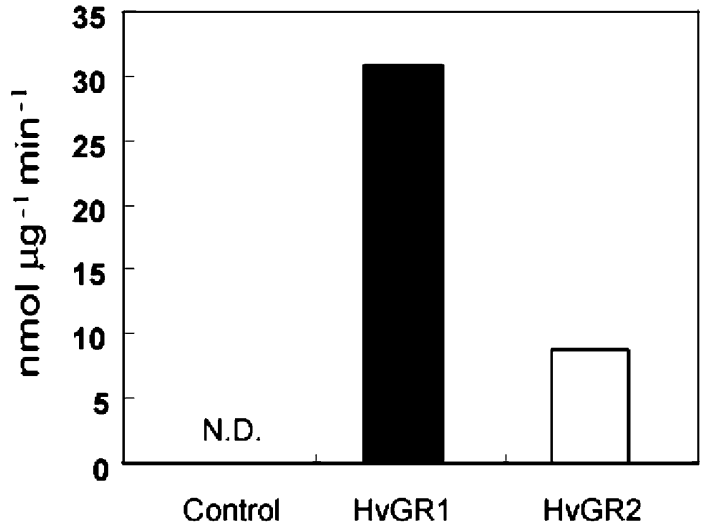

Fig. 3 GR activity of recombinant HvGR1 and HvGR2. The recombinant AB063249 protein lacking a FAD-binding domain and part of the NADPH-binding domain was used as a control

as a probe, microarray analysis showed that $O s G R l$ is upregulated under Fe-deficient conditions (data not shown).

Northern blot analysis using the $H v G R l$ probe detected two bands ( $\sim 2$ and $\sim 1.5 \mathrm{~kb}$; Fig. $4 \mathrm{~b})$. The smaller fragment may have been the partial sequence of $H v G R 1$. Only one band representing a $\sim 2-\mathrm{kb}$ fragment was detected using $H v G R 2$ as a probe.
GR activity increases in response to Fe-deficiency

Total protein was extracted from Fe-sufficient and Fe-deficient barley, wheat, rice, and maize to check the GR activity in response to $\mathrm{Fe}$-deficiency. GR activity increased in barley $(\times 1.85)$, wheat $(\times 1.24)$, and in maize $(\times 3.01)$ root tissues with Fe-deficiency (Table 1). The barley and wheat results are in line with those of the Northern blot analysis, whereas GR activity slightly decreased in rice roots $(\times 0.87)$. GR activity increased in rice $(\times 1.52)$, barley $(\times 1.27)$, and maize $(\times 2.95)$ shoot tissues. These results correspond with the Northern blot analyses for wheat, barley, and rice. We did not detect a band for maize, likely because we lacked a maize-specific probe. This means that we do not know whether these genes are post-transcriptionally regulated in maize. The enzyme activity was higher in root tissue than shoot tissue for all species examined.

\section{Discussion}

We cloned the genes for chloroplastic (HvGR1) and cytosolic ( $H v G R 2)$ GR in barley. GR is a ubiquitous enzyme that has been cloned from a number of prokaryotic and 
Fig. 4 Northern blot analysis for GR1 and GR2 in graminaceous plants.

(a) Northern blot analysis for chloroplastic GR (GRI). HvGRI was used as a probe.

(b) Northern blot analysis for cytosolic GR (GR2). HvGR2 was used as a probe for barley and wheat. OsGR2 was used as a probe for rice
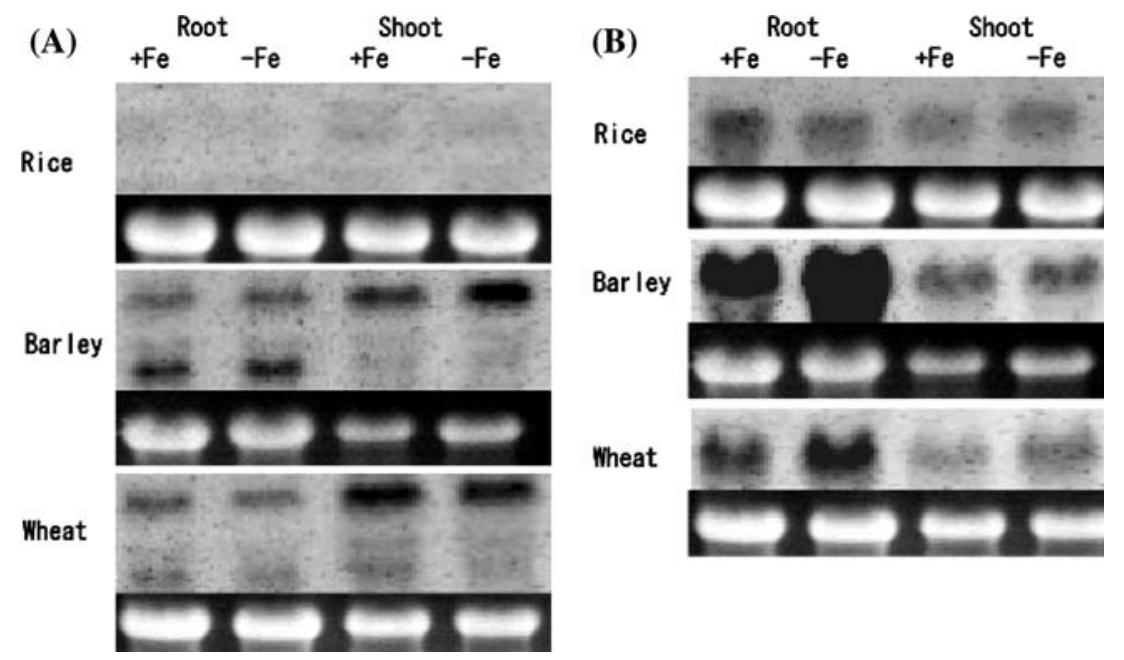

eukaryotic species (e.g., Greer and Perham 1986; Edwards et al. 1990; Foyer et al. 1991, Creissen et al. 1992). The sequences of cytosolic and chloroplastic GR are conserved among graminaceous crops (Fig. 1) and it appears that the expression of $G R$ is regulated by similar biotic and abiotic stresses. The $H v G R 2$ and $T a G R 2$ genes were mainly expressed in root tissue and a weak band was observed in shoot tissue. Similar expression patterns were observed for OsGRl in that expression was mainly observed in root and calli, rather than in leaves (Kaminaka et al. 1998). Conversely, the expression of $P s G R 2$ was very low in root tissue and weak in shoots and flowers (Stevens et al. 1997; Romero-Puertas et al. 2006). These results suggest that the expression of graminaceous and non-graminaceous GR may be regulated differently. It has also been reported that the expression of $P s G R 1$ and $P s G R 2$ are regulated differentially in response to various stresses such as mechanical wounding and increased temperature (Romero-Puertas et al. 2006). In barley, both $H v G R 1$ and $H v G R 2$ were upregulated by Fe-deficiency, whereas wheat exposed to the same conditions only exhibited increased levels of TaGR2 (Fig. 4).

Although we did not examine the expression of $G R$ in response to abiotic stresses other than Fe-deficiency, studies using the differential display protocol showed that the expression of $H v G R 2$ is upregulated under salt stress
(Ueda et al. 2002). GR specific activity was also measured to determine whether this enzyme is regulated post-transcriptionally. The ZmGRl is post-transcriptionally regulated in bundle sheath cells (Pastori et al. 2000), as are PsGRI and $P s G R 2$ in response to various abiotic stresses (RomeroPuertas et al. 2006). We observed increased GR activity in Fe-deficient barley, wheat, and maize roots and Fe-deficient rice, barley, and maize leaves. The expression of $H \nu G R 1$ was mainly localized to shoot tissues and that of $H v G R 2$ to root tissues. Therefore, it may be concluded that the increased GR activity in shoot tissue was due to the upregulation of $H v G R 1$, whereas the increase in root tissue may have been a result of the upregulation of $H v G R 2$. Northern blot analyses for barley and wheat confirmed this hypothesis by revealing that the upregulation of gene expression is directly proportional to the increase in GRspecific activity. Moreover, the expression and enzyme activity for $O s G R 2$ decreased in response to Fe-deficiency. These results suggest that $G R 1$ and $G R 2$ genes are not posttranscriptionally regulated in rice, wheat, or barely. We did not detect clear bands for maize using Northern blot analysis, likely because $H v G R 1$ and $H v G R 2 / O s G R 2$ were used as probes. The GR specific activity, however, clearly showed that the gene is upregulated in Fe-deficient maize root and shoot tissues.

Table 1 Glutathione reductase activity in response to Fe-deficiency stress

\begin{tabular}{|c|c|c|c|c|c|c|}
\hline & \multicolumn{3}{|l|}{ Root } & \multicolumn{3}{|l|}{ Shoot } \\
\hline & $+\mathrm{Fe}$ & $-\mathrm{Fe}$ & Ratio & $+\mathrm{Fe}$ & $-\mathrm{Fe}$ & Ratio \\
\hline Rice & $125 \pm 14.1$ & $109 \pm 5.2$ & 0.87 & $52 \pm 0.3$ & $79 \pm 0.3$ & 1.52 \\
\hline Barley & $150 \pm 17.6$ & $278 \pm 5.1$ & 1.85 & $51 \pm 0.3$ & $65 \pm 0.6$ & 1.27 \\
\hline Wheat & $117 \pm 11.4$ & $145 \pm 6.8$ & 1.24 & $75 \pm 0.6$ & $76 \pm 0.3$ & 1.00 \\
\hline Maize & $36 \pm 04.5$ & $110 \pm 0.3$ & 3.01 & $21 \pm 0.3$ & $62 \pm 1.4$ & 2.95 \\
\hline
\end{tabular}

The values represent reduction of nmol GSSG mg ${ }^{-1}$ crude extract $\min ^{-1}$. The values followed by represent the SD. The samples were processed at least in duplicate 
GR may allow plants to cope with Fe-deficiency in a variety of ways. Redox systems using heme and non-heme proteins such as cytochromes, catalase, peroxidases, and ferredoxin require Fe. Thus, under Fe-deficiency stress, the activity of these enzymes declines. GSH scavenges ROS via the Asc-GSH cycle and glutathione peroxidase (GPX). Although the Asc-GSH cycle is downregulated under Fedeficient conditions (Zaharieva and Abadía 2003), given that the activity of SOD increases (Tewari et al. 2005), GR may play a role in coping with Fe-deficiency-induced oxidative stress through the GPX cycle in combination with SOD (Fig. 5). It should be noted that the oxidative stress induced by Fe-deficiency is fundamentally different from that induced by salt or heavy metal stress, whereby the production of ROS as well as the activities of catalase and peroxidases increase (Patra and Panda 1998; Kim et al. 2005). Conversely, under conditions of Fe-deficiency, the production of ROS does not increase significantly, but rather, oxidative stress occurs as the activities of some enzymes involved in scavenging ROS decrease.

GR may also play a role in internal Fe homeostasis. Mobilization of Fe may help plants cope with Fe-deficiency stress. Graziano and Lamattina (2005) and Graziano et al. (2002) recently discussed the role of $\mathrm{NO}$ in $\mathrm{Fe}$ mobilization in plants. Interestingly, the ability of NO to induce Fe mobilization is dependent on GSH while that of chelators is independent of GSH (Watts and Richardson 2001). This suggests that NO alone does not have the capacity to remove $\mathrm{Fe}$ from intermediates and that it may require the reducing capacity of GSH. Alternatively, or in combination with this latter mechanism, GSH may form a mixed Fe complex with $\mathrm{NO}$ in order to acquire the appropriate lipophilicity and charge to diffuse or be

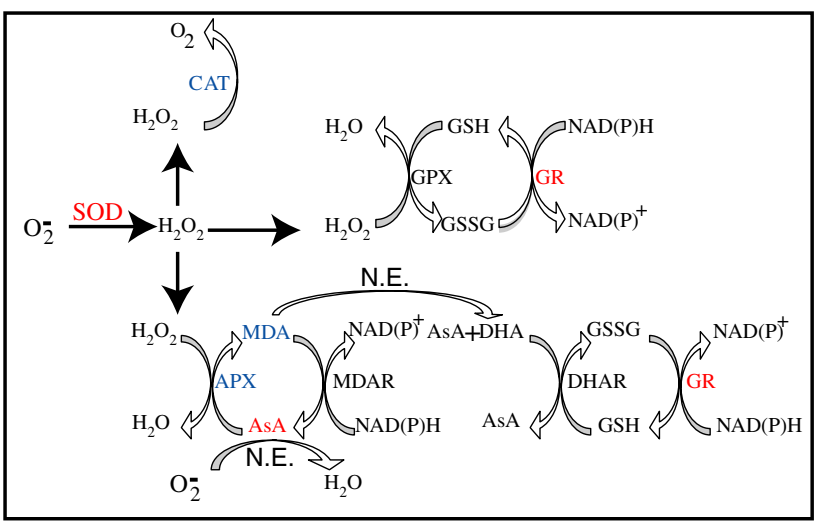

Fig. 5 The role of glutathione in coping with Fe-deficiency-induced oxidative stress. CAT, catalase; GPX, glutathione peroxidase; APX, ascorbate peroxidase; MDA, monodehydroascorbate; AsA, ascorbic acid; MDAR, MDA reductase; DHA, dehydroascrobate; DHAR, DHA reductase; N.E., non-enzymatic reaction. The activity/amount of the enzymes/compounds shown in red increases under Fe-deficiency, while that of shown in blue decreases transported from the cell (Watts and Richardson 2002). The presence of dithiol dinitrosyl-Fe complexes within cells has been previously demonstrated (Vanin 1991). As part of this complex, GSH may play an important role in signal transduction for the regulation of gene expression in response to different stresses and Fe-homeostasis. However, a S-nitrosylated form of GSH has been suggested to act as a transport molecule for NO, thereby increasing its half-life and allowing for effective biological activity (Lipton et al. 2001; Lipton 2001). GSH is also essential for the activity of glutathione S-transferase, which specifically interacts with the dinitrosyl-diglutathionyl-Fe complex and behaves like a storage protein for this complex in vivo and in vitro (Maria et al. 2003; Turella et al. 2003). These results suggest that GR may play a role in internal $\mathrm{Fe}$ homeostasis in graminaceous plants and thereby allow plants to cope with Fe-deficiency.

Acknowledgements We thank Ms Yuko Ogo and Dr Motofumi Suzuki for supporting our experiments and Dr Yasuhiro Ishimaru for useful discussions.

\section{References}

Alfenito M, Souer E, Goodman CD, Buell R, Mol J, Koes R, Walbot V (1998) Functional complementation of anthocyanin sequestration in the vacuole by widely divergent glutathione S-transferases. Plant Cell 10:1135-1149

Apel K, Hirt H (2004) Reactive oxygen species: metabolism, oxidative stress, and signal transduction. Annu Rev Plant Biol 55:373-399

Bashir K, Inoue H, Nagasak S, Takahashi M, Nakanishi H, Mori S, Nishizawa NK (2006) Cloning and characterization of Deoxymugineic acid synthase genes in graminaceous plants. J Biol Chem 281:32395-32402

Bradford MM (1976) A rapid and sensitive method for the quantification of microgram quantities of protein utilizing the principle of protein-dye-binding. Anal Biochem 72:248-254

Brunold C, Rennenberg H (1997) Regulation of sulfur metabolism in plants: first molecular approaches. Prog Bot 58:164-186

Cakmak I, Marschner H (1988) Enhanced superoxide radical production in roots of zinc-deficient plants. J Exp Bot 39:1449-1460

Chen Y, Barak P (1982) Iron nutrition of plants in calcareous soils. Adv Agron 35:217-240

Cobbett CS (2000) Phytochelatins and their roles in heavy metal detoxification. Plant Physiol 123:825-832

Creissen G, Edwards EA, Enard C, Wellburn A, Mullineaux P (1992) Molecular characterization of glutathione reductase cDNAs from pea (Pisum sativum L.). Plant J 2(1):129-131

Curie C, Briat JF (2003) Iron transport and signalling in plants. Ann Rev Plant Biol 54:183-206

Edwards E, Rawsthorne S, Mullineaux P (1990) Subcellular distribution of multiple forms of glutathione reductase in leaves of pea (Pisum sativum L.). Planta 180:278-284

Engler-Blum G, Meier M, Frank J, Muller GA (1993) Reduction of background problems in nonradioactive northern and Southern blot analyses enables higher sensitivity than 32P-based hybridizations. Anal Biochem 210:235-244. 10.1006/abio.1993.1189

Foyer CH, Lelandais M, Galap C, Kunert K-J (1991) Effects of elevated cystosolic glutathione reductase activity on the cellular 
glutathione pool and photosynthesis in leaves under normal and stress conditions. Plant Physiol 97:863-872

Graziano M, Lamattina L (2005) Nitric oxide and iron in plants: an emerging and converging story. Trends Plant Sci 10:4-8

Graziano M, Beligni MV, Lamattina L (2002) Nitric oxide improves internal iron availability in plants. Plant Physiol 130:1852-1859

Greer S, Perham RN (1986) Glutathione reductase from Escherichia coli: cloning and sequence analysis of the gene and relationship to other flavoprotein disulfide oxidoreductases. Biochemistry $25: 2736-2742$

Halliwell B, Gutteridge JM (1986) Oxygen free radicals and iron in relation to biology and medicine: some problems and concepts. Arch Biochem Biophys 246(2):501-514

Higuchi K, Suzuki K, Nakanishi H, Yamaguchi H, Nishizawa NK, Mori S (1999) Cloning of nicotianamine synthase genes, novel genes involved in the biosynthesis of phytosiderophores. Plant Physiol 119:471-479

Higuchi K, Watanabe S, Takahashi M, Kawasaki S, Nakanishi H, Nishizawa NK, Mori S (2001) Nicotianamine synthase gene expression differs in barley and rice under Fe-deficient conditions. Plant J 25:159-167

Ishikawa T, Madhusudhan R, Shigeoka S (2003) Effect of iron on the expression of ascorbate peroxidase in Euglena gracilis. Plant Sci 165:1363-1367

Iturbe-Ormaetxe I, Moran JF, Arrese-Igor C, Gogorcena Y, Klucas RV, Becana M (1995) Activated oxygen and antioxidant defences in iron-deficient pea plants. Plant Cell Environ $18: 421-429$

Kaminaka H, Morita S, Nakajima M, Masumura T, Tanaka K (1998) Gene cloning and expression of cytosolic glutathione reductase in rice (Oryza sativa L.). Plant Cell Physiol 39(12):1269-1280

Kanazawa K, Higuchi K, Nishizawa NK, Fushiya S, Chino M, Mori S (1994) Nicotianamine aminotransferase activities are correlated to the phytosiderophore secretions under Fe-deficient conditions in Gramineae. J Exp Bot 45(281):1903-1906

Kim SY, Lim J-H, Park MR, Kim YJ, Park TI, Seo YW, Choi KG, Yun SJ (2005) Enhanced antioxidant enzymes are associated with reduced hydrogen peroxide in barley roots under saline stress. J Biochem Mol Biol 38:218-224

Lipton SA (2001) Nitric oxide and respiration. Nature 413:118-121

Lipton AJ, Johnson MA, Macdonald T, Lieberman NW, Gozal D, Gaston B (2001) S-nitrosylothiols signal ventilatory response to hypoxia. Nature 430:171-174

Ma JF, Taketa S, Chang Y-C, Takeda K, Matsumoto H (1999) Biosynthesis of phytosiderophores in several Triticeae species with different genomes. J Exp Bot 50:23-726

Maria FD, Pedersen JZ, Caccuri AM, Antonini G, Turella P, Stella L, Bello ML, Federici G., Ricci G (2003) The specific interaction of dinitrosyl-diglutathionyl-iron complex, a natural NO carrier, with the glutathione transferase superfamily. J Biol Chem 278:42283-42293

Marrs KA (1996) The functions and regulation of glutathione Stransferase in plants. Annu Rev Plant Physiol Plant Mol Biol 47:127-158

Meister A (1988) Glutathione metabolism and its selective modification. J Biol Chem 263:17205-17208

Mori S (2001) The role of mugineic acid in iron acquisition: progress in cloning the genes for transgenic rice. In: Ae N, Arihara J, Okada K, Srinivasan A (eds) Plant nutrition acquisition. Springer-Verlag, Tokyo, pp 120-139

Mullineaux PM, Creissen GP (1997) Glutathione reductase: regulation and role in oxidative stress. In: Scandalios JG (ed) Oxidative stress and the molecular biology of antioxidants. Cold Spring Harbor Laboratory Press, Cold Spring Harbor, NY, USA, pp $667-713$
Noctor G, Foyer CH (1998) Simultaneous measurement of foliar glutathione $\gamma$-glutamylcysteine and amino acids by high-performance liquid chromatography: comparison with two other assay methods for glutathione. Anal Biochem 264:98-110

Noctor G, Gomez L, Vanacker H, Foyer CH (2002) Interactions between biosynthesis, compartmentalization and transport in the control of glutathione homeostasis and signaling. J Exp Bot 53:1283-1304

Ogawa K, Tasaka Y, Mino M, Tanaka Y, Iwabuchi M (2001) Association of glutathione with flowering in Arabidopsis thaliana. Plant Physiol 42:524-530

Pastori GM, Mullineaux PM, Foyer CH (2000) Post-transcriptional regulation prevents accumulation of glutathione reductase protein and activity in the bundle sheath cells of maize1. Plant Physiol 122:667-675

Patra J, Panda B (1998) A comparison of biochemical response to oxidative and metal stress in seedlings of barley, Hordeum vulgare L. Environ Pollut 101:99-105

Romero-Puertas MC, Corpas FJ, Sandalio LM, Marina L, RodríguezSerrano M, del Río LA, Palma JM (2006) Glutathione reductase from pea leaves: response to abiotic stress and characterization of the peroxisomal isozyme. New Phytol 170:43-52

Stevens GR, Creissen GP, Mullineaux PM (1997) Cloning and characterization of a cytosolic glutathione reductase cDNA from pea (Pisum sativum L.) and its expression in response to stress. Plant Mol Biol 35:641-654

Takagi S (1976) Naturally occurring iron-chelating compounds in oatand rice-root washing. I. Activity measurement and preliminary characterization. Soil Sci Plant Nutr 22:423-433

Tewari RK, Kumar P, Neetu, Sharma PN (2005) Signs of oxidative stress in the chlorotic leaves of iron starved plants. Plant Sci 169:1037-1045

Turella P, Pedersen JZ, Caccuri AM, Maria FD, Mastroberardino P, Bello ML, Federici G, Ricci G (2003) Glutathione transferase superfamily behaves like storage proteins for dinitrosyl-diglutathionyl-iron complex in heterogeneous systems. J Biol Chem 278:42294-42299

Ueda A, Shi W, Nakamura T, Takabe T (2002) Analysis of saltinducible genes in barley roots by differential display. J Plant Res 115:119-130

Vanin AF (1991) Endothelium-derived relaxing factor is a nitrosyl iron complex with thiol ligands. FEBS Lett 289:1-3

Vernoux T, Wilson RC, Seeley KA, Richheld JP, Muroy S, Brown S, Maughan SC, Cobbett CS, VanMontagu M, Inzè D, May MJ, Sung ZR (2000) The root meristemless1/cadmium sensitive2 gene defines a glutathione dependent pathway involved in initiation and maintenance of cell division during postembryonic root development. Plant Cell 12:97-110

Watts RN, Richardson DR (2001) Nitrogen monoxide (NO) and glucose: unexpected links between energy metabolism and NOmediated iron mobilization from cells. J Biol Chem 276: 4724-4732

Watts RN, Richardson DR (2002) The mechanism of nitrogen monoxide (NO)-mediated iron mobilization from cells. Eur J Biochem 269:3383-3392

Yoshihara T, Kobayashi T, Goto F, Masuda T, Higuchi K, Nakanishi H, Nishizawa NK, Mori S (2003) Regulation of Fe-deficiency responsive gene, Ids2 of barley in tobbaco. Plant Biotech 20: $33-41$

Zaharieva TB, Abadía J (2003) Iron deficiency enhances the levels of ascorbate, glutathione, and related enzymes in sugar beet roots. Protoplasma 221:269-275

Zaharieva TB, Gogorcena Y, Abadía J (2004) Dynamics of metabolic responses to iron deficiency in sugar beet roots. Plant Sci 166:1045-1050 